\title{
91. NUEVAS APORTACIONES AL CONOCIMIENTO DE LA FLORA DEL MACIZO DE LA SIERRA DE LÍBAR (MÁLAGA-CÁDIZ). II.
}

\author{
Manuel BECERRA PARRA
}

\begin{abstract}
New records for the flora of the Sierra de Líbar (Grazalema Natural Park, Málaga-Cádiz provinces)
Palabras clave. Flora, Parque Natural Sierra de Grazalema, Málaga-Cádiz, Sierra de Líbar.

Key words. Flora, Natural Park, Grazalema, Málaga-Cádiz, Sierra de Líbar.
\end{abstract}

Durante este año hemos continuado con nuestras herborizaciones en el Macizo de la Sierra de Líbar con el fin de completar el catálogo florístico de este conjunto montañoso situado entre las provincias de Málaga y Cádiz. Fruto de estas recolectas son 34 citas nuevas para la Sierra de Líbar, de las cuales 9 lo son para el Parque Natural Sierra de Grazalema, 23 para la zona malagueña de dicho parque natural y dos para la provincia de Cádiz. Además hemos localizado una nueva población de Erodium recoderi, geraniácea endémica del Sector Rondeño, de la que hasta la fecha sólo se conocían 5 localidades y que consideramos que debería ser catalogada En Peligro de Extinción. Todo el material se encuentra depositado en el herbario de la Universidad de Málaga (MGC).

\section{Isoetes durieui Bory}

MÁLAGA. Montejaque. Los Cucaderos. TF 9569.780 m.s.m. Arroyuelo, areniscas. M. Becerra; A. Rivas. MGC 53.584 .

Primera cita para el Macizo de la Sierra de Líbar de esta especie catalogada como Vulnerable por el Catálogo Andaluz de Flora Silvestre de Andalucía (Blanca et al., 2000). Únicamente hemos podido localizar una población formada por unos 50 individuos y que ocupa unos $5 \mathrm{~m}^{2}$. Juncales enanos (Junco pygmaei-Isoetetum velati) (Pérez Latorre et al., 1996).

\section{Cheilanthes guanchica Bolle}

MÁlaGA. Montejaque. Los Cucaderos. $30 \mathrm{~S}$ TF 9670.760 m.s.m. grietas húmedas y umbrosas de roquedos, areniscas del Aljibe. 20.02.03. M. Becerra; A. Rivas. MGC 53.523.

Primera cita para el P.N. Sierra de Grazalema. La población localizada está formada por escasos individuos. Forma parte de comunidades relícticas de Mucizonia hispida y Cheilantes guanchica (Pérez Latorre et al., 1996).

\section{Cheilantes maderensis Lowe}

MÁLAGA. Benaoján. Sierra de Benaoján, Cañada de Montejaque. TF 9967.570 m.s.m. Grietas de rocas umbrías, margocalizas, exposición sureste. 18.01.03. M. Becerra. MGC 55.374.

Primera cita para el P.N. Sierra de Grazalema. La población localizada, formada por escasos individuos aislados, presenta una ecología distinta a la descrita para la especie, rocas ácidas (Cabezudo \& Salvo in Valdés et al.1987).

Athyrium filix-femina (L.) Roth

MÁLAGA. Montejaque. Los Cucaderos, Arroyo de Foreila. TF 9669. 800 m.s.m. Márgenes de un arroyo, areniscas. 28.08.03 M. Becerra; F. Sánchez-Tundidor. MGC 55.372.

Primera cita para el P.N. Sierra de Grazalema. Sólo hemos localizado una población fragmentada en dos núcleos, siendo el número de individuos encontrados de cinco.

\section{Polystichum setiferum (Forsskal) Woynar}

MÁlaGA. Benaoján. Sierra de Juan Diego, Hoyo de la Ventana. TF 9665. 1270 m.s.m. Fondo de una sima, calizas. 07.12.02. M. Becerra; J. Amaya. MGC 55.394.

Primera cita para la Sierra de Líbar. Sólo hemos localizado una única población en la zona cacuminal de la Sierra de Juan Diego formada por 
tres individuos y que ocupa una superficie de $1 \mathrm{~m}^{2}$.

\section{Arenaria armerina Bory subsp. armerina}

CÁDIZ. Villaluenga del Rosario. Sierra de los Pinos, Pico de los Pinos, caídas hacia Garganta Barrida. TF 8756.1.360 m.s.m. Claros de un piornal, calizas. 10.07.2003. M. Becerra. MGC 55.395.

Según flora Ibérica (López, 1990) esta cariofilácea no estaba presente en la provincia de Cádiz por lo que es la primera cita para esta provincia. Especie localizada en los claros del piornal existente en la cumbre de la Sierra de los Pinos junto a Erysimum rondae Polatschek, Arenaria arundana Gallego, Clypeola jonthlaspi subsp. microcarpa (Moris) Arcangeli, Iberis carnosa subsp. granatensis (Boiss. \& Reuter) Moreno, Valeriana tuberosa L., etc.

\section{Lavatera olbia L.}

MÁLAGA. Cortes de la Frontera. Sierra de los Pinos, Cañada del Panderete. TF 8854. 960 m.s.m. Márgenes de un arroyo, margocalizas. 16.04.03. M. Becerra; C. Guerrero. MGC 55393.

Primera cita para la zona malagueña del P.N. Sierra de Grazalema. Sólo hemos podido localizar un individuo. Claros de un matorral de Calicotome villosa.

Drosophyllum lusitanicum (L.) Link

MÁLAGA. Cortes de la Frontera. Base de la Sierra de los Pinos. TF 8556.720 m.s.m. Cortafuegos, areniscas. 27.07.03. M. Becerra. MGC 55.360 .

Primera cita de esta especie, protegida en Andalucía bajo la figura de Vulnerable (Blanca, G. et al., 2000), para la sierra de Líbar. La población localizada está formada por unos 300 individuos. $\mathrm{Al}$ parecer también se encuentra en el pinar del Pimpollar, Cortes de la Frontera. Aparece en claros de un brezal-aulagar (Genisto tridentisStauracanthetum boivinii quercetosum lusitanicae) (Pérez Latorre et al., 1996).

Arabidopsis thaliana (L.) Heynh.

MÁlAGA. Montejaque. Loa Cucaderos, Arroyo Foreila. TF 9669. 800 m.s.m. Pastizal sobre suelos rezumantes, areniscas. 04.05.03. M. Becerra. MGC 55.368.

Primera cita para la zona malagueña del P.N. Sierra de Grazalema. Aparece en comunidades terofíticas anfibias de Loto subbifloriChaetopogonetum fasciculati (Pérez Latorre et al., 1996).

Iberis carnosa Willd. subsp. granatensis (Boiss. \& Reuter) Moreno

MÁLAGA. Cortes de la Frontera. Sierra de los Pinos, Pico de los Pinos. $30 \mathrm{~S}$ TF 8756. 1.340 m.s.m. Roquedo, calizas, norte. 09.V.03. M. Becerra; C. Guerrero. MGC 53.877.

Primera cita para la zona malagueña del P.N. Sierra de Grazalema. Aparece en suelos pobres procedentes de la descomposición de dolomías, en los claros del piornal de la zona cacuminal de la Sierra de los Pinos junto a Erysimum rondae Polatschek, Arenaria arundana Gallego, Clypeola jonthlaspi subsp. microcarpa (Moris ) Arcangeli, Valeriana tuberosa L., etc.

Coronopus didymus (L.) Sm.

MÁLAGA. Benaoján. La Vega. Tf 9966. 520 m.s.m. Márgenes de cultivos, depósitos aluviales. 07.04.03. M. Becerra. MGC 55.370.

Primera cita para esta especie cosmopolita en el P.N. Sierra de Grazalema. Comunidades nitrófilas.

Moricandia moricandioides (Boiss.) Heywood

MÁLAGA. Jimera de Líbar. Carretera de Benaoján, por encima de las Artezuelas. TF 9660.520 m.s.m. Márgenes de la carretera, margocalizas. 19.05.03. M. Becerra. MGC 55.359.

Primera cita para la zona malagueña del P.N. Sierra de Grazalema.

\section{Pistorinia breviflora Boiss.}

MÁLAGA. Montejaque. Los Cucaderos. TF 9669. 780 m.s.m. Grietas de rocas, areniscas. 08.06.03. M. Becerra. MGC 55.358.

Primera cita para la Sierra de Líbar. Aparece formando parte de pastizales terofíticos.

\section{Sanguisorba hybrida (L.) Nordborg}

MÁLAGA. Montejaque. El Quejigal, Cañada de los Mulos. TF 9569. 800 m.s.m. Márgenes de un arroyo, areniscas. 08.06.03. M. Becerra. MGC 55.357.

Primera cita para la zona malagueña del P.N. Sierra de Grazalema. Sotobosque de quejigales (Rusco-Quercetum canariensis quercetosum broteroi) (Pérez Latorre et al., 1996). 


\section{Pyrus bourgeana Decne}

MÁLAGA. Montejaque. La Calera. UF 0070. Roquedo, calizas. 10.08.03. M. Becerra. MGC 55.361 .

Primera cita para la zona malagueña del P.N. Sierra de Grazalema. Sólo hemos podido localizar un único individuo en un lapiaz calizo, presentando una ecología distinta a la descrita para la especie, ya que es acidófila.

\section{Prunus insititia L.}

MÁlAGA. Montejaque. Tavizna. UF 0069. 700 m.s.m. bosquete con Acer monspessulanum, Crataegus monogyna, etc., calizas. 12.10.02. M. Becerra; J. Amaya. MGC 52841.

Primera cita en la Sierra de Líbar para esta rosácea protegida en Andalucía como vulnerable (Blanca, G. et al., 2000). Forma parte de acerales (Daphno latifoliae-Aceretum granatensis) (Pérez Latorre et al., 1998).

\section{Anthyllis cytisoides L.}

MÁLAGA. Jimera de Líbar. Carretera de Cortes, junto al mirador. $30 \mathrm{~S}$ TF 9458.460 m.s.m. Talud sobre la carretera, margocalizas. 07.IV.03. M. Becerra. MGC 53.881.

Primera cita para la zona malagueña del P.N. Sierra de Grazalema. Etapas de degradación de un encinar termomediterráneo (Smilaci mauritanicaeQuercetum rotundifoliae).

\section{Erodium recoderi Auriault \& Guittoneau}

MÁLAGA. Montejaque. Sierra de Algarrobo, Cancho de la Ermita. 30 S TF 9968. 700 m.s.m. Paredón calizo, noroeste. M. Becerra; J. Amaya. 05.IV.03. MGC 53.880.

Nueva cita para este endemismo rondense cuya área de distribución se restringe a las sierras de Grazalema y Líbar. La población localizada estaba formada por unos 50 individuos que florecen y fructifican bien, observándose regeneración. Son especies acompañantes Silene andryalifolia Pomel, Lavatera marítima Gouan, Saxifraga bourgeana Boiss. \& Reuter, etc.

\section{Erodium cheilanthifolium Boiss.}

CÁDIZ. Villaluenga del Rosario. Sierra de los Pinos, Pico de los Pinos. 30 STF 8756. Paredón calizo umbrío, exposición norte. 1.380 m.s.m. 10.V.03. M. Becerra; F. Sánchez Tundidor; A. Rivas.
MGC 53. 878.

Primera cita de esta geraniácea para el P.N. Sierra de Grazalema y la provincia de Cádiz. La única población localizada está formada por más de 300 individuos. Aparece en roquedos junto con Erysimum rondae Polatschek, Clypeola jonthlaspi subsp. microcarpa (Moris) Arcangeli, Linaria platycalyx Boiss., Biscutella sempervirens L., etc

Jasione foliosa subsp. minuta (Schultes) Font Quer MÁlAGA. Benaoján. Sierra del Palo, Los Canchos Negros. TF 9562. 1.240 m.s.m. Paredones umbríos, exposición norte, dolomías. 17.07.03. M. Becerra. MGC 55.356.

Primera cita para la zona malagueña del P.N. Sierra de Grazalema, donde sólo se conocía en los paredones de la cara norte de la Sierra del Pinar a más de $1.500 \mathrm{~m}$. de altitud. Hemos localizado una única población, formada por escasos individuos, que crece en paredones umbríos en exposición norte, junto a Draba hispanica, Erinus alpinus, Crepis albida, Saxifraga globulifera, etc.

\section{Nepeta apulei Ucria ex Guss}

MÁLAGA. Benaoján. Carretera de Cortes, por encima del Parral. TF 9762. 600 m.s.m. Base de un paredón calizo. 16.06.02. M. Becerra. MGC 52.790.

Primera cita para la zona malagueña del P.N. Sierra de Grazalema. La población localizada está formada por 5 individuos. Cerca de la Cueva de la Pileta, en el Cerro de la Pileta encontramos otra localidad compuesta por 10 individuos. Son especies acompañantes Klasea pinnatifida (Cav.) Cass., Centaurea melitensis L., Leuzea conifera (L.) DC, etc.

Anarrhinum laxiflorum Boiss.

MÁLAGA. Cortes de la Frontera. Sierra de los Pinos, Vereda del Romeral. TF 9056. 840 m.s.m. Pastizal, dolomías. 16.04.03. M. Becerra; C. Guerrero. MGC 55.371.

primera cita para la zona malagueña del P.N. Sierra de Grazalema. Pastizales anuales sobre dolomías (Arenarietum arundanae) (Pérez Latorre et al., 1998).

\section{Sibthorpia europaea L.}

MÁlAGA. Montejaque. Los Cucaderos, Arroyo de Foreila. TF 9669. 800 m.s.m. Márgenes de un arroyo, areniscas. 28.08.03 M. Becerra; F. 
Sánchez-Tundidor. MGC 55.373.

Primera cita para el P.N. Sierra de Grazalema. Para la provincia de Málaga, sólo teníamos constancia de un pliego herborizado en Casares (MGC 22.636) por lo que, ésta sería la segunda cita provincial. Aparece muy abundante en los arroyos de las zonas con areniscas del noroeste del término municipal de Montejaque, muy probablemente esté en la zona de Pimpollar (Cortes de la Frontera), al suroeste del Macizo.

Hymenostemma pseudoanthemis (G. Kunze) Willk. MÁlAGA. Los Cucaderos, bajando desde Piedra Amarilla hacia el Arroyo de Foreila, junto a la linde del Quejigal. 04.V.03. M. Becerra. MGC 55.369 .

Primera cita de esta asterácea, catalogada en Andalucía como Vulnerable (Blanca, G. et al., 2000), para el Macizo de la Sierra de Líbar. la única población localizada hasta la fecha está formada por más de 300 individuos. Aparece en pastizales terofíticos silicícolas de Tuberaria guttata L.

\section{Merendera androcymbiodes Valdés}

MÁLAGA. Cortes de la Frontera. Llanos de Líbar, cerca de la Fuente de Líbar. 30 S TF 9261. 980 m.s.m. Pastizal, margocalizas. M. Becerra; F. Sánchez Tundidor; C. Guerrero. MGC 53.583.

Primera cita para la zona malagueña del P. N. Sierra de Grazalema de éste endemismo bético restringido a las sierras de Ronda, Grazalema, Subbética de Córdoba y Líbar. La población localizada está formada por uno 100 individuos. Pastizales de Hordeum bulbosum L. (GaudinioHordeion bulbosi) (Pérez Latorre et al., 1998).

Tulipa sylvestris L. subsp. australis (Link) Pamp. MÁLAGA. Cortes de la Frontera. Sierra de los Pinos, Cerros del Espino. $30 \mathrm{~S}$ TF 8957. 1.140 m.s.m. Roquedo, calizas. 10.V.03. M. Becerra; A. Rivas; F. Sánchez Tundidor. MGC 53.879.

Es la primera cita para la zona malagueña del P.N. Sierra de Grazalema. La población está formada por unos 30 individuos.

\section{Narcissus fernadesii G. Pedro}

MÁLAGA. Benaoján. Llanos de Líbar, Fuente de Líbar. 30 S TF 9261.980 m.s.m. Pastizal sobre suelos encharcados, margocalizas. 05.III.03. MGC 53.881 .
Primera cita para esta amarilidácea, catalogada en Andalucía como Vulnerable (Blanca, G. et al., 2000), en la zona malagueña del P.N. Sierra de Grazalema. La población está formada por más de 1000 individuos. También hemos localizado otras dos localidades, El Pantano y Llano del Mures, Montejaque. La primera localidad está compuesta por más de 1000 individuos y la segunda por unos 10. Pastizales de Hordeum bulbosum L. y juncales basófilos nitrófilos sobre suelos encharcados temporalmente (Ranunculo macrophylli-Juncetum inflexi) (Pérez Latorre et al., 1998).

Narcissus bugei (Fernández Casas) Fernández Casas MÁLAGA. Montejaque. Los Cucaderos, arroyo Foreila. 30s TF 9569. 760 m.s.m. Pastizal en los márgenes de un arroyo, suelos rezumantes, areniscas del Aljibe. 20.02.03. M. Becerra; A. Rivas. MGC 53.476.

Primera cita de este endemismo bético, catalogado como En Peligro de Extinción en Andalucía (Blanca, G. et al., 1999), para el P.N. Sierra de Grazalema. La población localizada, dividida en tres núcleos que no superan los 14 individuos, es la única que hasta el presente se conoce para el Sector Aljíbico. Se encuentra gravemente amenazada por la presión ganadera, especialmente de vacas, que consumen las flores no dejando que fructifiquen. Juncal en los claros de un alcornocal y un brezal-jaral con Cistus ladanifer L., Stauracanthus boivinii (Webb) Samp., Lavandula stoechas L., Erica australis L., etc.

\section{Narcissus $x$ dubius Gouan}

MÁLAGA. Benaoján. Cerro de la Pileta. $30 \mathrm{~S}$ TF 9763. 680 m.s.m. pastizal, margocalizas. 02.II.03. M. Becerra. MGC 53.883.

Primera cita para el P.N. Sierra de Grazalema de este híbrido entre Narcissus assoanus Dufour subsp. praelongus A. Barra \& López y Narcissus papyraceus $\mathrm{L}$. En la población encontrada, formada por 7 individuos, aparece conviviendo con el primero de los parentales.

\section{Iris pseudoacorus L.}

MÁLAGA. Montejaque. Los Cucaderos. $30 \mathrm{~S}$ TF 9670. 720 m.s.m. Laguna, areniscas. 04.V.03. M. Becerra. MGC 53.882.

Primera cita para la zona malagueña del P.N. Sierra de Grazalema. También tenemos constancia 
de que existe otra población en el cercano río Gaduares. Pequeña laguna de aguas permanentes junto a Ranunculus hederaceus L., Ranunculus ophioglossifolius Vill., Callitriche stagnalis Scop., Veronica anagallis-aquatica L., etc. (Ranunculo hederacei-Callitrichetum stagnalis) (Pérez Latorre et al., 2002).

\section{Aceras anthropophorum (L.) W.T. Aiton}

MÁlAGA. Benaoján. Sierra del Palo, Pilas del Tunio. TF 9461. 1.300 m.s.m. Pedregal, calizas. 25.V.01. M. Becerra. MGC 52.659.

Primera cita para la zona malagueña del P.N. Sierra de Grazalema. La población está formada por dos núcleos que no superan los 20 individuos. Claros de un lastonar de Festuca scariosa (Lag.) Ascherson \& Graebner.

\section{Neotinea maculata (Desf.) Stearn}

MÁLAGA. Montejaque. El Quejigal, Cañada de los Mulos. Tf 9569. 820 m.s.m. Márgenes de un arroyo, areniscas. 01.05.03. M. Becerra. MGC 55.363 .

Primera cita para la zona malagueña del P.N. Sierra de Grazalema. Sotobosque de quejigales (Rusco-Quercetum canariensis quercetosum broteroi) (Pérez Latorre et al., 1996). También la hemos localizado sobre calizas en el Mures (Montejaque), Sierra del Palo (Benaoján) y Sierra de los Pinos (Cortes de la Frontera).

\section{Ophrys dyris Maire}

MÁLAGA. Montejaque. Los Calabazales. Tf 9769.690 m.s.m. Pastizal sobre suelos rezumantes, arcillas. 04.05.03. M. Becerra. MGC 55.362.

Primera cita para el P.N. Sierra de Grazalema. Sólo hemos localizado una población formada por 10 individuos. Pastizal sobre suelos encharcados junto a Ophrys lutea Cav., Serapias parviflora Parl., Sisymbrella aspera (L.) Spach.

\section{BIBLIOGRAFÍA}

APARICIO, A. y S. SILVESTRE - 1996- Guía de la Flora del Parque Natural Sierra de Grazalema. Consejería de Medio Ambiente. Junta de Andalucía.

BLANCA, G., B. CABEZUDO, J.E. HERNÁNDEZBERMEJO, C.M. HERRERA, J. MUÑOZ y B.
VALDÉS -1999- Libro Rojo de la Flora Silvestre Amenazada de Andalucía. Especies En Peligro. Consejería de Medio Ambiente. Junta de Andalucía.

BLANCA, G., B. CABEZUDO, J.E. HERNÁNDEZBERMEJO, C.M. HERRERA, J. MUÑOZ y B. VALDÉS -1999- Libro Rojo de la Flora Silvestre Amenazada de Andalucía. Especies Vulnerables. Consejería de Medio Ambiente. Junta de Andalucía.

CABEZUDO, B. y A.E. SALVO-1987- Cheilanthes in Valdés et al. (eds.). Flora Vascular de Andalucía Occidental 1:49-51. Editorial Ketres. Barcelona.1987.

LÓPEZ GONZÁLEZ, G. -1990- Arenaria in Castroviejo, S. et al. (eds.). Flora Ibérica 2:172224. CSIC. Madrid.

PÉREZ LATORRE, A. V., A. GALÁN DE MERA, U. DEIL y B. CABEZUDO -1996-. Fitogeografía y vegetación del sector Aljíbico (Cádiz-Málaga, SW de España). Acta Bot. Malacitana 21: 241267.

PÉREZ LATORRE, A.V., P. NAVAS, D. NAVAS, Y. GIL y B. CABEZUDO -1998- Datos sobre la flora y vegetación de la Serranía de Ronda (Málaga, España). Acta Bot. Malacitana 23: 149-191.

PÉREZ LATORRE, A. V., P. NAVAS, D. NAVAS, Y. GIL y B. CABEZUDO -2002- Datos sobre la flora y la vegetación de la cuenca del río Guadiamar (Sevilla-Huelva, España). Acta Bot. Malacitana 27: 189-228.

Aceptado para su publicación en octubre de 2003

Dirección del autor. Dpto. Biología Vegetal. Facultad de Ciencias. Universidad de Málaga. Campus de Teatinos. 28071, Málaga. 